\title{
Comportamiento en la hemodinamia en pacientes con hemorragia controlada por monitor ultrasónico (USCOM)
}

\author{
Marco Antonio Brito Espinosa, ${ }^{*}$ Carlos Alberto Cortes Soto, ${ }^{*}$ Felipe de Jesús Montelongo, ${ }^{*}$ \\ María Magdalena Reyes Pérez, ${ }^{*}$ Javier Corona Muñoz*
}

\section{RESUMEN}

Introducción: La hemodinamia es la parte de la biofísica que se encarga del estudio anatómico y funcional del corazón, de la dinámica de la sangre en el interior de las estructuras sanguíneas, así como de la mecánica del corazón. Objetivo: Comparar la hemodinamia con el dispositivo no invasivo USCOM antes y después de presentar hemorragia controlada.

Material y métodos: Se realizó un estudio tipo observacional, prospectivo, longitudinal y comparativo en pacientes de entre 16 y 65 años de edad en un periodo de seis meses como fecha corte para este premio académico (marzo de 2016-proyecto aún en curso).

Resultados: Se obtuvieron promedios de las diferentes variables hemodinámicas, de precarga, postcarga e inotropismo, observando cambios tempranos a la exanguinación de los pacientes, siendo principalmente las resistencias vasculares sistémicas las que se modifican.

Discusión: Existen cambios hemodinámicos tempranos a la exanguinación de pequeñas cantidades de sangre en los pacientes, cambios determinados por el sistema USCOM, sistema fácil de usar, no invasivo y preciso en los resultados ofrecidos.

Conclusiones: En este estudio podemos observar cómo las variables relacionadas a la postcarga (resistencias vasculares sistémicas) son las primeras variables que se modifican, por lo que con la pérdida de pequeñas cantidades de sangre llegamos a observar esto.

Palabras clave: Hemodinamia, gasto cardiaco, variabilidad de volumen sistólico, poder cinético, índice de inotropismo.

\section{SUMMARY}

Introduction: Hemodynamics is the part of biophysics that is responsible for the anatomical and functional study of the heart, the dynamics of blood inside the blood structures as well as the mechanics of the heart.

Objective: To compare the hemodynamics of our patients with the non-invasive device USCOM, before and after presenting with controlled hemorrhage.

Material and methods: An observational, prospective, longitudinal and comparative study was performed in patients between the ages of 16 and 65 in a period of 6 months as a cutoff date for this academic award. (March 2016-ongoing). Results: We obtained averages of the different hemodynamic variables, both preload, afterload and inotropism, observing early changes to the exanguination of the patients, being mainly the systemic vascular resistances that are modified. Discussion: There are early hemodynamic changes to the exanguination of small amounts of blood in the patients, changes determined by the USCOM system, system easy to use, non-invasive and accurate results offered.

Conclusions: In this study we can observe how the variables related to afterload (systemic vascular resistance) are the first variables that are modified, so that with the loss of small amounts of blood we get to observe this.

Key words: Hemodynamics, cardiac output, systolic volume variability, kinetic power, inotropic index.

\section{RESUMO}

Introdução: A hemodinâmica é a parte da biofísica responsável pelo estudo anatômico e funcional do coração, da dinâmica do sangue dentro das estruturas sangüíneas, bem como a mecânica do coração.

Objetivo: Comparar a hemodinâmica com o dispositivo USCOM não invasivo, antes e após a apresentação da hemorragia controlada.

Material e metodos: Foi realizado um estudo observacional, prospectivo, longitudinal e comparativo em pacientes entre 16 e 65 anos de idade, em um período de 6 meses, como data limite para este prêmio acadêmico (Março de 2016 - projeto ainda em andamento).

Resultados: Obtivemos as médias das diferentes variáveis hemodinâmicas, tanto de pré-carga, pós-carga e inotropismo, observando as alterações precoces na exanguinação dos pacientes, sendo principalmente as resistências vasculares sistêmicas aquelas que são modificadas.

\footnotetext{
* ISEM Las Américas, Ecatepec.
}

Recepción: 01/09/2017. Aceptación: 13/07/2018.

Este artículo puede ser consultado en versión completa en http://www.medigraphic.com/medicinacritica
Discussão: Existem alterações hemodinâmicas precoces na exsanguinação de pequenas quantidades de sangue nos pacientes, mudanças determinadas pelo sistema USCOM fáceis de usar, não invasivas e precisas nos resultados oferecidos.

Conclusões: Neste estudo podemos observar como as variáveis relacionadas à pós-carga (resistência vascular sistêmica) são as primeiras variáveis que são modificadas,

de modo que com a perda de pequenas quantidades de sangue podemos observar isso.

Palavras-chave: Hemodinâmica, debito cardíaco, variabilidade do volume sistólico, potência cinética, índice de inotropismo.

\section{INTRODUCCIÓN}

La Medicina del Enfermo en Estado Crítico es una subespecialidad que en nuestros tiempos es esencial para el funcionamiento de los centros hospitalarios, ya que se encarga de manejar a los pacientes que ya no pueden seguir siendo tratados en áreas generales de hospitalizacion: ${ }^{1}$ los enfermos hospitalizados en los que no se tiene un pronóstico fatal inherente a su patología de base o a su estado de gravedad inicial, en los que, a pesar del manejo estándar para el proceso patológico original, han alcanzado un estado de pérdida de la homeostasis grave, que lleva a la presencia de la disfunción orgánica, que los hace requirentes de soporte externo para mantener la vida, siendo así pacientes candidatos al área de terapia intensiva; área especializada del hospital que tendrá que contar con la infraestructura y el personal especializado que pueda mantener esa función a costa de un soporte de vida. Este manejo tendrá el objetivo de dar tiempo al paciente y permitir, una vez que sea tratado el factor desencadenante, que la función orgánica se recupere o llevar al enfermo a un estado de homeostasis que le permita permanecer con vida, manejo que intenta evitar que la disfunción orgánica sea sumatoria a otros sistemas hasta llegar a la disfunción de múltiples órganos y la muerte del paciente.

Este estudio está enfocado en una pequeña parte del mundo tan vasto de la medicina crítica.

Enfocándonos ya en el tema a tratar de nuestro estudio, mencionamos que para la medicina crítica es esencial, por medio del monitoreo cardiovascular avanzado, determinar de manera fidedigna la causa por la que el enfermo desarrolló pérdida de la homeostasis. Además, una vez implementado el manejo específico de soporte, es muy importante poder monitorizar en tiempo real los cambios posteriores para tratar de llegar a metas preestablecidas. 
Sin duda, poder determinar la hemodinamia del paciente da información de gran relevancia al médico de la terapia intensiva para poder tomar decisiones de vitalidad para sus pacientes.

La hemodinamia (del griego, aĩ $\mu \alpha$ (haima) sangre

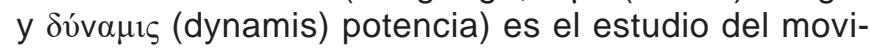
miento de la sangre a través del sistema vascular. Es difícil imaginar cómo serían los conceptos actuales de las enfermedades críticas sin los enormes conocimientos fisiológicos y anatómicos derivados de los últimos 60 años de estudio de ésta. André Cournand comentó al recibir el Premio Nobel en 1956, «el catéter cardiaco fue la llave en la cerradura». Al «girar esta llave», Cournand y cols. nos han llevado a una nueva era en la comprensión de la función cardiaca normal y la fisiopatología de las enfermedades críticas de los seres humanos. Hace tan sólo cuatro décadas, Swan y Ganz introdujeron a la práctica clínica un catéter terminado en un balón inflable en el que el flujo sanguíneo permite llevar la punta hasta la arteria pulmonar.

Hoy en día, la medición rutinaria de presiones, flujos y resistencias en las diferentes cámaras del corazón y lechos sistémico y pulmonar permite la objetivación del compromiso hemodinámico de diferentes condiciones fisiopatológicas y definir si el paciente se encuentra en estado de choque y qué tipo de choque es, logrando discernir entre un estado de choque hiperdinámico y uno hipodinámico.

El estado de choque hiperdinámico se refiere al hecho de que el paciente mantiene un gasto cardiaco dentro de parámetros normales o altos, pero que existe otra causa que hace que el gasto cardiaco sea insuficiente para alcanzar un aporte de oxígeno adecuado; situación que en la mayoría de las ocasiones se presenta por una pérdida de la función de la mecánica vascular, que hace que las resistencias vasculares sistémicas se encuentren por debajo de los límites de la normalidad, llevando al enfermo a un estado de vasodilatación importante. Estos mecanismos son específicos del estado de choque anafiláctico y del estado de choque séptico. ${ }^{2}$ En este último, además de los mecanismos ya referidos, también puede presentarse una disfunción mitocondrial y de la microcirculación, que llevan a una falla metabólica y a una disminución en el número de capilares para el intercambio y utilización de oxígeno. ${ }^{3}$

El estado de choque hipodinámico se refiere al hecho de que el paciente desarrolló esta patología debido a una disminución en el gasto cardiaco, situación que se presenta por una disminución en el volumen de eyección del ventrículo izquierdo, que puede ser secundaria a tres procesos: una disfunción de la bomba cardiaca, denominándose, si éste es el caso, como choque cardiogénico; a una disminución en el volumen intravascular, estado denominado como choque hipovolémico, y, por último, a una obstrucción al flujo de sangre del ventrículo derecho o a un proceso de compresión externa del corazón que no permite que haya un llenado cardiaco adecuado, ambas situaciones denominadas como estado de choque obstructivo. ${ }^{3}$

En este contexto, la monitorización cardiovascular avanzada con la medición del gasto cardiaco sería una medida más fidedigna para poder realizar esta clasificación de manera correcta. Se requiere, sin lugar a dudas, esta medida tanto como medida directa como para poder inferir las resistencias vasculares sistémicas. El hecho de que no se realice adecuadamente este diagnóstico diferencial puede llevar a errores en el manejo del paciente que resulten peligrosos.

Una vez que se realiza el diagnóstico específico de la causa del estado de choque, se tienen que implementar medidas terapéuticas para poder llevar al paciente a la remisión del proceso de hipoperfusión tisular. En este contexto, igualmente la medida del gasto cardiaco se convierte en una meta terapéutica a alcanzar. ${ }^{4,5}$

\section{Gasto cardiaco, definición e historia}

La medida del gasto cardiaco se refiere a la cantidad de sangre que sale de los ventrículos del corazón a la circulación mayor o menor, según sea el caso del ventrículo izquierdo o derecho, por unidad de tiempo (casi siempre referida en un minuto); por lo tanto, sus unidades de medición serán los litros por minuto (L/min), la fórmula ampliamente descrita en textos de la literatura médica se describe como sigue: ${ }^{4,5}$

$$
\begin{gathered}
\mathrm{GC}=\mathrm{VEV} \times \mathrm{FC} \\
\mathrm{GC}=\text { Gasto cardiaco } \\
\mathrm{VEV}=\text { Volumen de eyección ventricular } \\
\text { (por latido cardiaco) } \\
\mathrm{FC} \mathrm{=} \mathrm{Frecuencia} \mathrm{cardiaca} \mathrm{(latidos} \mathrm{por} \mathrm{minuto)}
\end{gathered}
$$

La técnica de medición de gasto cardiaco fue descrita por primera vez en 1870 por Adolf Fick, ${ }^{4,6}$ quien calculó el gasto cardiaco de un animal utilizando el consumo de oxígeno y las concentraciones de oxígeno en muestras de sangre arterial y venosa. ${ }^{4,7,8}$

$$
\begin{gathered}
\mathrm{GC}=\mathrm{VO}_{2} /\left(\mathrm{CaO}_{2}-\mathrm{CvO}_{2}\right) \\
\mathrm{GC}=\text { Gasto cardiaco } \\
\mathrm{vO}_{2}=\text { Consumo de oxígeno } \\
\mathrm{CaO}_{2}=\text { Contenido de oxígeno arterial } \\
\mathrm{CvO}_{2}=\text { Contenido de oxígeno venoso }
\end{gathered}
$$

En 1897, Stewart describió experimentos con el principio de dilución de indicadores, inyectó cloruro de sodio en la circulación venosa central de animales y midió posteriormente su concentración en la arteria femoral. ${ }^{9}$ Hamilton modificó este principio para dar cuenta de las concentraciones variables de inyectado diluido en 
el tiempo, desarrollando una curva de concentración a través del tiempo para reflejar este fenomeno. ${ }^{7}$ Pudo demostrar, entonces, que el gasto cardiaco fue igual a la cantidad de colorante indicador (verde de indocianina) inyectado, dividido por la integral del área bajo la curva de dilución medida en el lado arterial, conocida hoy como la ecuación de Stewart-Hamilton. ${ }^{9}$

$$
\text { Flujo }=(\mathrm{Ci} \mathrm{Vi}) / \int \mathrm{C}(\mathrm{t}) \mathrm{dt}
$$

$\mathrm{Ci}=$ Concentración inicial del inyectado

$\mathrm{Vi}=$ Volumen inicial de inyección

$$
\int=\text { Integral }
$$

$\mathrm{C}(\mathrm{t})=$ Concentración de inyectado diluido en el tiempo $\mathrm{dt}=$ Área bajo la curva de la dilución

Basándose en el mismo concepto de la dilución de indicadores, Fegler introdujo la termodilución en 1954, inyectando una solución fría como indicador y midiendo los cambios en la temperatura sanguínea detectados distalmente. ${ }^{10}$ En 1970, Swan et al. desarrollaron lo que denominaron un catéter de lumen múltiple dirigido hacia la arteria pulmonar, por medio de un globo de flotación, denominado actualmente como catéter de Swan-Ganz o catéter arterial pulmonar (CAP). ${ }^{11}$ La introducción de este dispositivo permitió a los médicos medir el gasto cardiaco por termodilución.

Después de 50 años, este método todavía se considera el estándar de oro clínico para la medición del gasto cardiaco. ${ }^{4}$

En la actualidad, existe una variedad muy amplia de métodos tanto invasivos como no invasivos para poder realizar una medición del gasto cardiaco. ${ }^{4,5}$ Para fines de nuestro estudio nos enfocaremos en dos métodos no invasivos que describiremos de manera detenida.

\section{Medición del gasto cardiaco por evaluación ecocardiográfica Doppler y utilización de la ecuación de continuidad}

La medición del gasto cardiaco (GC) mediante el uso de dispositivos basados en la tecnología Doppler ha experimentado un crecimiento exponencial en los últimos años debido, fundamentalmente, a su no invasividad respecto al catéter arterial pulmonar y a la introducción, lenta, pero imparable, de la ecocardiografía en la unidad de cuidados intensivos como medio diagnóstico y como monitor hemodinámico. ${ }^{5}$

\section{Fundamentos físicos}

La aplicación de ultrasonidos en el rango de 2-10 MHz con capacidad de interactuar con los tejidos humanos (en nuestro caso, su capacidad de reflexión sobre los eritrocitos en movimiento) constituye la base física para la determinación ecocardiográfica del gasto cardiaco por medio de la utilización de la ecuación de continuidad, principio físico que de igual forma utiliza el monitor USCOM. ${ }^{5}$

La ley de Doppler expresa la relación matemática existente entre el cambio aparente que se produce en la frecuencia de una onda de ultrasonido cuando el emisor y/o el receptor se encuentran en movimiento relativo. La formulación matemática es la siguiente:5,12

$$
\begin{gathered}
\Delta \mathrm{f}=(2 \mathrm{ft}(\mathrm{v} / \mathrm{c}))(\cos \theta) \\
\Delta \mathrm{f}=\text { Cambio de frecuencia. } \\
\mathrm{ft}=\text { Frecuencia original transmitida } \\
\mathrm{v}=\text { Velocidad relativa entre emisor y receptor } \\
\mathrm{c}=\text { Velocidad del sonido en el medio (en los tejidos } \\
\text { humanos blandos se toma como media } \\
1.54 \mathrm{~m} / \mathrm{s}) \\
\cos \theta=\text { Coseno del ángulo de incidencia } \\
\text { entre emisor y receptor. }
\end{gathered}
$$

A nivel práctico, esta propiedad nos sirve para, mediante un transductor que es capaz de generar y detectar ultrasonidos, enviar un haz de ultrasonidos de $\mathrm{ft}$ conocida a nivel cardiovascular (tracto de salida del ventrículo izquierdo o derecho, aorta ascendente y descendente), que en parte chocarán contra los eritrocitos en movimiento y volverán a ser detectados a una frecuencia distinta con la que fueron enviados, con lo que podrá calcularse $\Delta f$. Si tenemos en cuenta que además conocemos el valor de $c$ y el valor de $\cos \theta$ es un valor fijo en algunos dispositivos y en otros puede calcularse (dadas las características matemáticas de la función coseno, se consideran clínicamente aceptables hasta $\left.30-40^{\circ}\right) \cdot{ }^{5,12}$

La única variable que nos queda por conocer es $v$ que podremos despejar:

$$
v=\Delta f(c / 2) f t \cos \theta
$$

Dicha $v$ es la velocidad de los eritrocitos en movimiento y, en consecuencia, la velocidad del flujo sanguíneo. 5,12

Existen dos modos distintos de aplicar el efecto Doppler al estudio de la velocidad del flujo sanguíneo que nos proporcionarán informaciones distintas. Tanto si se usa el Doppler continúo como el pulsado, el análisis matemático mediante integración (cálculo del área bajo la curva) a lo largo de un ciclo cardiaco nos dará como resultado lo que se conoce como integral velocidad-tiempo (IVT) en centímetros. Esta medida representa la longitud de una columna de sangre que pasa por un punto determinado durante un ciclo cardiaco. Posteriormente, asumiendo que el punto donde calculamos el espectro de velocidad posee determinadas características geométricas, que habitualmente se asume como circular en el sistema cardiovascular (anillo 
aórtico), podremos calcular el área de su sección mediante su medición directa, asumiendo una geometría circular por medio de la siguiente fórmula:5,12-15

$$
\begin{gathered}
\pi \mathrm{r} 2 \\
\pi=3.1416 \\
\mathrm{r}=\text { Radio del diámetro total medido }
\end{gathered}
$$

Con todas estas variables se podrá calcular a continuación el volumen sistólico y el gasto cardiaco con la ecuación de continuidad:5,12-15

$$
\begin{gathered}
\mathrm{GC}=((\pi \mathrm{r} 2)(\mathrm{IVT})(\mathrm{FC})) / 1000 \\
\mathrm{GC}=\mathrm{Gasto} \text { cardiaco en litros por minuto } \\
\pi=3.1416 \\
\mathrm{r}=\text { Radio del área seccional }
\end{gathered}
$$

IVT = Integral de velocidad tiempo en centímetros

$\mathrm{FC}=$ Frecuencia cardiaca en latidos por minuto

Se puede, además, hacer una simplificación de la fórmula sustituyendo el valor de $\pi$ de la siguiente manera: ${ }^{13-15}$

$$
\begin{gathered}
\mathrm{GC}=((\pi(\mathrm{D} / 2) 2)(\mathrm{IVT})(\mathrm{FC})) / 1000 \\
\mathrm{GC}=((\mathrm{D} 2 \pi / 22)(\mathrm{IVT})(\mathrm{FC})) / 1000 \\
\mathrm{GC}=(\mathrm{D} 2(3.1416 / 4))(\mathrm{IVT})(\mathrm{FC})) / 1000 \\
\mathrm{GC}=((\mathrm{D} 20.785)(\mathrm{IVT})(\mathrm{FC})) / 1000 \\
\text { Monitor ultrasónico de gasto cardiaco } \\
\text { (USCOM) }
\end{gathered}
$$

Recientemente, se ha centrado mucho interés en los dispositivos de medición no invasiva de gasto cardiaco que utiliza tecnología Doppler. Para que un dispositivo de este tipo sea útil clínicamente debe ser preciso, claro y ser capaz de detectar cambios direccionales significativos, que reflejen la respuesta del corazón a una intervención, más comúnmente cambios en el volumen sistólico y gasto cardiaco. ${ }^{16,17}$ El monitor ultrasónico de gasto cardiaco (USCOM) es un dispositivo no invasivo de ultrasonido Doppler de onda continúa. Este dispositivo tiene una precisión medida por espectro Doppler de 2-3 Herz ${ }^{16,18}$ El transductor del dispositivo USCOM se coloca en el hueco supraesternal, para medir el gasto cardiaco de corazón izquierdo, o en la región precordial, para medir el gasto cardiaco de corazón derecho; es importante que el operador, por medio de la observación en la pantalla del dispositivo, dirija el transductor para obtener una imagen adecuada. ${ }^{19}$

El dispositivo detecta y grafica un espectro Doppler del flujo de la válvula pulmonar o aórtica. Para fines de nuestro estudio obtendremos la medición del gasto cardiaco del ventrículo izquierdo, por lo que nos enfocaremos en la obtención del espectro Doppler de flujo aórtico. Por medio del espectro obtenido, el monitor realiza la determinación de la integral de velocidad tiempo y de la frecuencia cardiaca por minuto. Para obtener de manera correcta el espectro Doppler, los fabricantes del monitor recomiendan realizar movimientos de manera sistematizada. ${ }^{15-19,20-29}$

De inicio, se requiere colocar el transductor en el hueco supraesternal, dibujando una línea paralela con respecto al esternón del paciente. Posteriormente, se realiza un movimiento de angulación dirigida hacia la cabeza del paciente, cuidando de no despegar por ningún motivo el transductor de la piel. Durante el proceso, el operador tiene que estar pendiente de la pantalla del monitor, observando las características del espectro Doppler dibujado. Inicialmente, se reconoce un perfil sistólico de baja velocidad y de duración sistólica corta. Este es el flujo de la aorta ascendente. ${ }^{19}$

Los movimientos de angulación deberán ser lentos para ir valorando al mismo tiempo las características del espectro Doppler en la pantalla del monitor. Al continuar inclinando el transductor, la velocidad del espectro aumentará a medida que el haz se alinea con la dirección del flujo aórtico, además, los lados del triángulo se volverán más rectos y tendrán más definición. El mejor ángulo de insonación será el que obtenga la mayor definición de ambos lados del espectro y la mayor velocidad de flujo. De principio probablemente la punta del triángulo del espectro será difusa y no bien definida. El siguiente paso es proceder a realizar movimientos de basculación del transductor entre las 4:00 y 8:00 horas en comparación con las manecillas de un reloj. Esto con el objetivo de mejorar la calidad de la imagen y así determinar una mejor área bajo la curva. ${ }^{19}$

El dispositivo cuenta con un software que puede también estimar por medio de la aplicación de ecuaciones de regresión indexadas a la altura del paciente, el diámetro de la sección transversal de la válvula aórtica. ${ }^{16,30}$ Una vez obtenidas estas tres mediciones, es capaz de calcular el gasto cardiaco con la fórmula de continuidad, ya referida con anterioridad, en el apartado de Doppler. ${ }^{13-15}$

EL monitor USCOM fue probado positivamente en estudios con catéteres de flujo en animales. ${ }^{16,31,32}$ Este monitor también ha sido comparado para su validación con el catéter de arteria pulmonar con resultados positivos. ${ }^{33}$ Dos estudios publicados recientemente por un grupo han sugerido que el USCOM en comparación con el monitor Doppler esofágico es capaz de realizar un seguimiento adecuado de los cambios en volumen sistólico después de intervenciones en los pacientes. ${ }^{34,35}$

\section{MATERIAL Y MÉTODOS}

Se realizó un estudio tipo observacional, prospectivo, longitudinal y comparativo. 


\section{Definición de las variables}

\section{Promedio de la integral de velocidad-tiempo:} cuantitativa. Definición operacional: promedio de tres ciclos cardiacos de la integral obtenida por el software del USCOM, después de hacer el trazado del espectro Doppler del flujo de sangre en sístole del tracto de salida del ventrículo izquierdo. Resultado: numérico en centímetros.

2. Frecuencia cardiaca: cuantitativa. Definición operacional: número de latidos por minuto del corazón de los pacientes participantes. Resultado: numérico en latidos por minuto.

3. Volumen sistólico: cuantitativa. Definición operacional: volumen de sangre que el corazón expulsa hacia la aorta durante el periodo de contracción (sístole). Resultado: numérico en mililitros.

4. Gasto cardiaco: cuantitativa. Definición operacional: medida del gasto cardiaco arrojada por la máquina de USCOM posterior a realizar la medición en hueco supraesternal de flujo aórtico y a agregar la edad, talla y peso de cada paciente al dispositivo. Resultado: numérico en litros/minuto.

5. Índice cardiaco: cuantitativo. Definición operacional: relación entre el volumen de sangre evacuado por el corazón cada minuto y los metros cuadrados de la superficie del cuerpo. Resultado: litros/ minuto $/ \mathrm{m}^{2}$.

6. Resistencias vasculares sistémicas: cuantitativo. Definición operacional: es la resistencia que ofrece el sistema vascular (excluida en este caso la circulación pulmonar) al flujo de sangre. Resultado: numérico en dinas $/ \mathrm{m}^{2}$.

7. Variabilidad de volumen sistólico: cuantitativo. Definición operacional: fenómeno natural por el que la presión del pulso arterial baja durante la inspiración y sube durante la espiración debido a las variaciones en la presión intratorácica secundarias a la ventilación con presión negativa. Resultado: numérico en milímetros de mercurio.

8. Índice de inotropismo: numérico. Definición operacional: hace referencia al potencial y a la energía cinética suministrada para poder realizar un latido de VI. Resultado: numérico en $\mathrm{W} / \mathrm{m}^{2}$.

9. Poder cinético (PKR): numérico. Definición operacional: cantidad de energía necesaria para poder desplazar un volumen en un área específica en una unidad de tiempo, manteniendo una adecuada relación flujo-presión.

Tamaño de la muestra: pacientes de entre 16 y 65 años que cumplieron con los estándares para poder donar sangre en un periodo del 01 de marzo de 2017 tomando la muestra hasta la fecha; sin embargo, el proyecto aún se encuentra en curso.
Muestra: no fue necesario realizar ningún tipo de muestra, ya que el universo de estudio pudo incluirse en su totalidad.

Criterios: fueron incluidos en el estudio todos los pacientes de entre 16 y 65 años, entre el periodo de 01 de marzo de 2017 hasta la fecha (sin embargo, el proyecto continua en curso), los cuales aceptaron participar, previa firma de consentimiento informado en el estudio.

Criterios de inclusión: Pacientes citados en el Banco de Sangre Estatal en el periodo comprendido desde el 01 de marzo del 2017 hasta la fecha, proyecto aún en curso. Pacientes de entre 16 y 65 años de edad. Pacientes que se encontraban sanos. Pacientes que aceptaron participar en el estudio, previa firma de carta de consentimiento informado. Pacientes en los que técnicamente fue realizable la medición de las variables con el monitor ultrasónico USCOM.

Criterios de exclusión (Anexo 1): Pacientes que no cumplieron los rangos de edad. Pacientes que no aceptaron participar en el estudio, previa firma de carta de consentimiento informado. Pacientes en los que técnicamente fue imposible realizar la medición técnica de las variables con el monitor ultrasónico USCOM.

Criterios de eliminación: Pacientes que en cualquier momento quisieron salir del estudio.

\section{RESULTADOS}

Una vez realizado el estudio y aplicados los criterios de inclusión, exclusión y eliminación en la población estudiada, se logró recabar la información de un total de 120 pacientes.

En las Figuras 1 a 4 pueden observarse los diferentes cambios en los parámetros hemodinámicos que se detectaron al inicio y al término de la exanguinación. Vemos cómo se elevan de forma importante variables de postcarga, precarga e inotropismo, cambios, que se

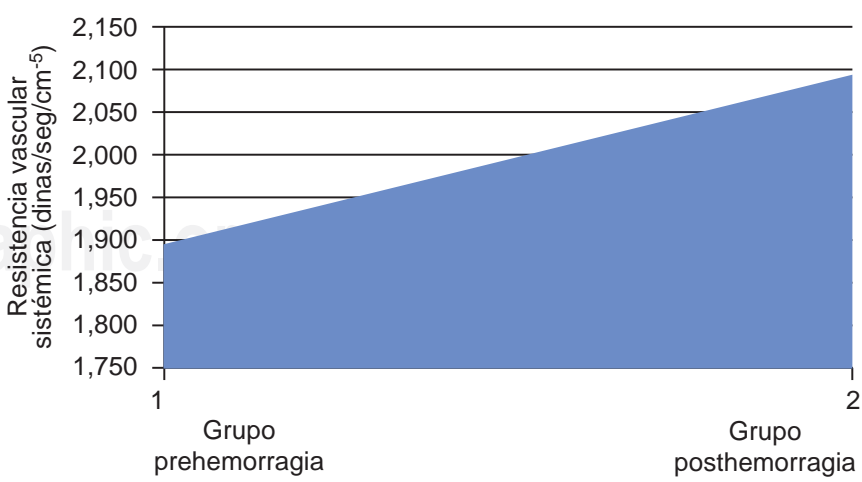

Fuente: Hoja de recolección de datos.

Figura 1: Se observan los cambios en la determinación de las resistencias vasculares por USCOM; vemos un aumento importante de las mismas al realizar el procedimiento de exanguiación en los pacientes en el Banco de Sangre. 
ven desde cifras de exanguinación aun menores a lo reportado en la literatura mundial $(750 \mathrm{~mL})$, por lo que los cambios en la hemodinamia son aun más tempranos. Las cifras en estos cambios son incluso menores de lo reportado y, sobre todo, el monitoreo con el sistema USCOM es efectivo, fácil de realizar y nos da amplia

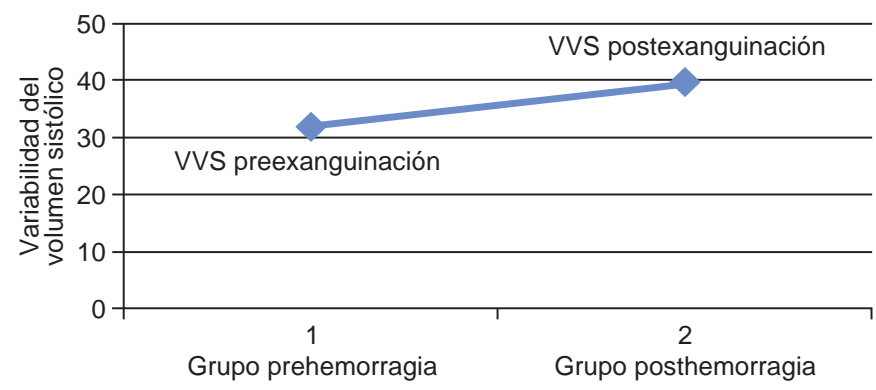

Fuente: Hoja de recolección de datos.

Figura 2: Se observa el cambio en los promedios al determinar la variabilidad de volumen sistólico; vemos un aumento en esta variable.

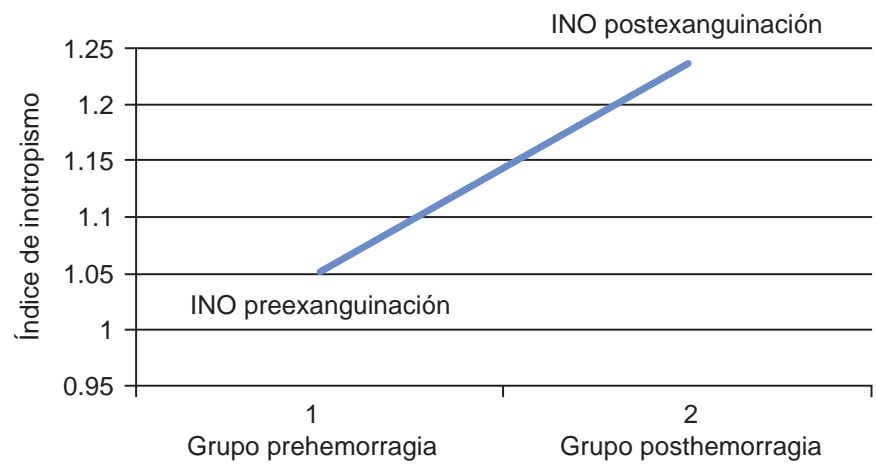

Fuente: Hoja de recolección de datos.

Figura 3: Se muestran los cambios en los promedios del índice de inotropismo antes y después de la hemorragia controlada; vemos como no hay aumento importante del mismo.

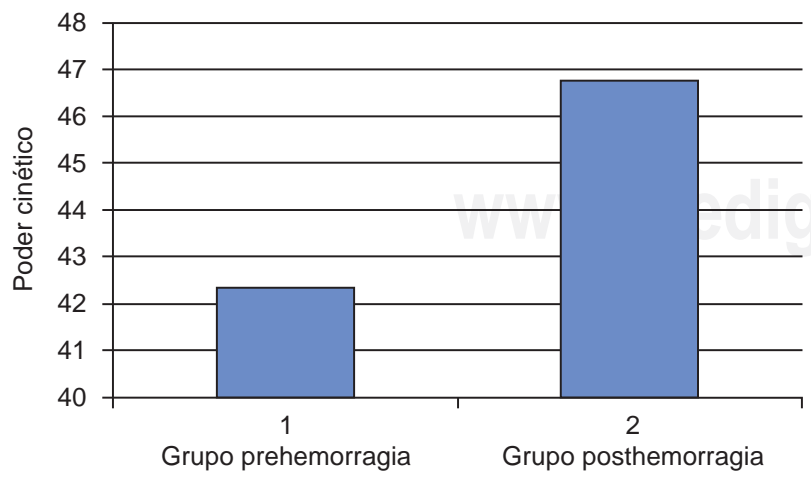

Fuente: Hoja de recolección.

Figura 4: Se comparan los diferentes promedios del poder cinético por USCOM; vemos que no hay un cambio de importancia en esta variable al realizar la hemorragia controlada. información precisa y eficaz para la toma de decisiones en bienestar de los pacientes.

\section{DISCUSIÓN}

En este estudio se decidió evaluar las diferentes variables hemodinámicas que determinan el USCOM al realizar una exanguinación, pretendiendo demostrar los diferentes cambios tempranos que puedan observarse con pérdidas hemáticas mínimas (450 mL). En las tablas antes colocadas pueden verse dichos cambios en los diferentes parámetros hemodinámicos con predominio en la postcarga, los cuales sin duda se detectaron con frecuencia, independientemente de la edad, género o factores indirectos. Además de que proporcionan amplia información de los cambios que pueden darse en la hemodinamia de forma temprana, son de gran ayuda para evaluar a los pacientes, pues nos dan la pauta para mejorar de forma inicial su estado general sin necesidad de dar soporte hasta que se presente el estado de choque.

\section{CONCLUSIONES}

Nuestro universo de estudio se compuso de pacientes que fueron aceptados y cumplieron los estándares para donar sangre en el Banco Estatal de Ecatepec. La distribución de género entre los pacientes fue principalmente en hombres.

Las medidas antropométricas de talla y peso en promedio refieren pacientes con complexión media que caen en el grupo de sobrepeso.

Nuestro objetivo principal es evaluar los diferentes cambios en las variables medidas con el monitor ultrasónico USCOM durante una hemorragia controlada (exanguinación de los pacientes en Banco de Sangre), logrando observar cuáles son los cambios que se presentan de forma temprana, considerando las variables para la precarga, postcarga o inotropismo, siendo esto lo más relevante que tomaremos en cuenta para tomar mejores decisiones que repercutan en el pronóstico de los pacientes.

En las terapias intensivas, actualmente ha disminuido el uso del estándar de oro clínico, lo que se explica tanto por su naturaleza invasiva como por el hecho de que no ha demostrado reducir la mortalidad. Por tal motivo, las actuales guías de práctica clínica para monitoreo hemodinámico sólo recomiendan este procedimiento para pacientes en estado de choque refractario y para pacientes con falla cardiaca derecha.

Las técnicas no invasivas de gasto cardiaco han cobrado importancia en los últimos años. En las guías de práctica clínica de monitoreo hemodinámico actuales se menciona a la ecocardiografía como la técnica inicial de elección tanto para la búsqueda del diagnóstico etioló- 
gico como para el seguimiento del monitoreo hemodinámico de los pacientes.

Sin duda, el monitor USCOM es una herramienta muy útil, fácil de realizar, no invasiva y con muchas opciones a favor para el monitoreo hemodinámico del paciente crítico, pero aún se necesita tener más evidencia al respecto de esta herramienta; sin embargo, en la bibliografía que utilizamos, los resultados fueron equiparables con el estándar de oro (catéter de flotación), por lo que consideramos que su uso es altamente recomendable.

En los resultados de este estudio puede verse claramente cómo las variables relacionadas con la postcarga (resistencias vasculares sistémicas) se ven aumentadas de forma más importante y más rápida, seguidas de las variables de precarga como la variabilidad de volumen sistólica, considerando que pueden observarse cambios importantes con la pérdida de pequeñas cantidades de sangre, cifras aun menores a las que se describen en la tabla de hemorragia para estado de choque determinado por los cirujanos, en la que se indican cifras aproximadas de $750 \mathrm{~mL}$, logrando incluso evaluar cambios con pérdidas de sólo $200 \mathrm{~mL}$. Es aún más factible observar cambios tempranos en los pacientes con el monitoreo por USCOM, pues es un método sencillo de utilizar, rápido y que otorga parámetros inmediatos.

Sin lugar a dudas, nuestro estudio adolece, en primer lugar, del hecho de contar con un número pequeño de pacientes y, en segundo, de haber sido realizado por un solo investigador (en estudios de procedimientos dependientes del operador es de utilidad medir la variabilidad interobservador).

Situación por la que esta afirmación no puede generalizarse a todos los pacientes en áreas críticas de todas las partes del mundo; sin embargo, nuestro estudio da pauta para que en el futuro haya una planeación de estudios multicéntricos con mayor número de pacientes que puedan confirmar si la tendencia de estos datos estadísticos persiste y puede ser replicable.

\section{BIBLIOGRAFÍA}

1. Miranda DR, Ryan DW, Schaufeli WB, Fidler V. Organisation and management of intensive care: a prospective study in 12 European countries. Berlin, Alemania: Springer Science \& Business Media, 2012.

2. Vincent JL, De Backer D. Circulatory shock. N Engl J Med. 2013;369(18):1726-1734. doi: 10.1056/NEJMra1208943.

3. Pinsky MR, Payen D. Functional hemodynamic monitoring. United Kingdom: Springer, 2016.

4. Lee AJ, Cohn JH, Ranasinghe JS. Cardiac output assessed by invasive and minimally invasive techniques. Anesthesiol Res Pract. 2011;2011:475151. doi: 10.1155/2011/475151.

5. Garcia X, Mateu L, Maynar J, Mercadal J, Ochagavia A, Ferrandiz A. Estimating cardiac output. Utility in the clinica practice. Available invasive and non-invasive monitoring. Med Intensiva. 2011;35(9):552-61. doi: 10.1016/j.medin.2011.01.014.

6. Prys-Roberts $\mathrm{C}$. The measurement of cardiac output. Brit $J$ Anaesth. 1969;41(9):751-760.
7. Reuter DA, Huang C, Edrich T, Shernan SK, Eltzschig HK. Cardiac output monitoring using indicator-dilution techniques: basics, limits, and perspectives. Anesth Analg. 2010;110(3):799811. doi: 10.1213/ANE.0b013e3181cc885a.

8. Kadota LT. Theory and application of thermodilution cardiac output measurement: a review. Heart Lung. 1985;14(6):605-616.

9. Stewart GN. Researches on the circulation time and on the influences which affect it. J Physiol. 1897;22(3):159-183.

10. Nishikawa T, Dohi S. Errors in the measurement of cardiac output by thermodilution. Can J Anaesth. 1993;40(2):142-153.

11. Swan HJ, Ganz W, Forrester J, Marcus H, Diamond G, Chonette D. Catheterization of the heart in man with use of a flow-directed balloon-tipped catheter. N Engl J Med. 1970;283(9):447-451.

12. Evans DH, McDicken WN. Doppler ultrasound. 2nd ed. New York: John Wiley and Sons. 2000.

13. Huntsman LL, Stewart DK, Barnes SR, Franklin SB, Colocousis JS, Hessel EA. Noninvasive Doppler determination of cardiac output in man. Clinical validation. Circulation. 1983;67(3):593602

14. Porter TR, Shillcutt SK, Adams MS, Desjardins G, Glas KE, Olson JJ, et al. Guidelines for the use of echocardiography as a monitor for therapeutic intervention in adults: a report from the American Society of Echocardiography. J Am Soc Echocardiogr. 2015;28(1):40-56. doi: 10.1016/j.echo.2014.09.009.

15. Beaulieu Y, Marik PE. Bedside ultrasonography in the ICU: part 1. Chest. 2005;128(2):881-895. doi: 10.1378/chest.128.2.881.

16. Hodgson LE, Forni LG, Venn R, Samuels TL, Wakeling GH. A comparison of the non-invasiveultrasonic cardiac output monitor (USCOM) with the oesophageal Doppler monitor during majorabdominal surgery. J Intensive Care Soc. 2016;17(2):103110. doi: $10.1177 / 1751143715610785$

17. Marik PE. Noninvasive cardiac output monitors: a state-of the-art review. J Cardiothorac Vasc Anesth. 2013;27(1):121-134. doi: 10.1053/j.jvca.2012.03.022.

18. Walker A, Olsson E, Wranne B, Ringqvist I, Ask P. Accuracy of spectral Doppler flow and tissue velocity measurements in ultrasound systems. Ultrasound Med Biol. 2004;30(1):127-132. doi: 10.1016/j.ultrasmedbio.2003.08.020.

19. USCOM Ltd. Available in: [http://www.uscom.com.au/]. Australia: The Association; c2000-2009 [Updated 2009; cited 2016 Nov 24]. USCOM Ltd; [About 55 screens]. Available in: http://www. talentmed.com.tw/download/UscomBasics.pdf

20. Soni NJ, Arntfield R, Kory P. Point-of-care ultrasound. Philadelphia: Elsevier Saunders, 2015

21. Crossingham IR Nethercott DR, Columb MO. Comparing cardiac output monitors and defining agreement: a systematic review and meta-analysis. J Intensive Care Soc. 2016;17(4):302-313. doi:10.1177/1751143716644457.

22. Eriksen M, Walloe L. Improved method for cardiac output determination in man using ultrasound Doppler technique. Med Biol Eng Comput. 1990;28(6):555-560.

23. Ihlen H, Amlie JP, Dale J, Forfang K, Nitter-Hauge S, Otterstad JE, et al. Determination of cardiac output by Doppler echocardiography. Br Heart J. 1984;51(1):54-60.

24. Sjöberg BJ1, Wranne B. Cardiac output determined by ultrasound-Doppler: clinical applications. Clin Physiol. 1990;10(5):463-473.

25. Rowland T, Obert P. Doppler echocardiography for the estimation of cardiac output with exercise. Sports Med. 2002;32(15):973-986

26. Lopes PC, Sousa MG, Camacho AA, Carareto R, Nishimori CT, Santos PS, et al. Comparison between two methods for cardiac output measurement in propofol anesthetized dogs: thermodilution and Doppler. Vet Anaesth Analg. 2010;37(5):401408. doi: 10.1111/j.1467-2995.2010.00552.x

27. Haites NE, McLennan FM, Mowat DH, Rawles JM. Assessment of cardiac output by the Doppler ultrasound technique alone. $\mathrm{Br}$ Heart J. 1985:53(2):123-129.

28. McLean AS, Needham A, Stewart D, Parkin R. Estimation of cardiac output by noninvasive echocardiographic techniques in the critically ill subject. Anaesth Intensive Care. 1997;25(3):250254.

29. Levitov A, Frankel HL, Blaivas M, Kirkpatrick AW, Su E, Evans D, et al. Guidelines for the appropriate use of bedside general and 
cardiac ultrasonography in the evaluation of critically ill patientspart II: cardiac ultrasonography. Crit Care Med. 2016;44(6):12061227. doi: $10.1097 /$ CCM.0000000000001847.

30. Nidorf SM, Picard MH, Triulzi MO, Thomas JD, Newell J, King ME, Weyman AE. New perspectives in the assessment of cardiac chamber dimensions during development and adulthood. J Am Coll Cardiol. 1992;19(5):983-938.

31. Critchley LA, Peng ZY, Fok BS, Lee A, Phillips RA. Testing the reliability of a new ultrasonic cardiac output monitor, the USCOM, by using aortic flowprobes in anesthetized dogs. Anesth Analg. 2005;100(3):748-753. doi: 10.1213/01. ANE.0000144774.42408.05.

32. Phillips RA, Hood SG, Jacobson BM, West MJ, Wan L, May $\mathrm{CN}$. Pulmonary artery catheter (PAC) accuracy and efficacy compared with flow probe and transcutaneous Doppler (USCOM): an ovine cardiac output validation. Crit Care Res Pract. 2012;2012:621496. doi: 10.1155/2012/621496.

33. Phillips R, Lichtenthal P, Sloniger J, Burstow D, West M, Copeland J. Noninvasive cardiac output measurement in heart failure subjects on circulatory support. Anesth Analg. 2009;108(3):881-886. doi: 10.1213/ane.0b013e318193174b.

34. Zhang J, Critchley LA, Huang L. Five algorithms that calculate cardiac output from the arterial waveform: a comparison with Doppler ultrasound. Br J Anaesth. 2015;115(3):392-402. doi: 10.1093/bja/aev254.

35. Huang L, Critchley LA. An assessment of two Doppler-based monitors to track cardiac output changes in anaesthetised patients undergoing major surgery. Anaesth Intensive Care. 2014;42(5):631-639.

\section{Correspondencia:}

Marco Antonio Brito Espinosa

2da Privada de Tlaxcala SN,

Col. Buenavista,

Mpio. Tultitlán, 54944, Estado de México.

E-mail: redlink_m@hotmail.com

\section{ANEXO}

Carta de consentimiento informado

Banco de Sangre Regional Las Américas,

Ecatepec de Morelos/Unidad de Cuidados intensivos

Fecha:

Nombre del paciente

(apellido paterno, apellido materno y nombre):

Folio/expediente

Se realizarán mediciones de vena cava, gasto cardiaco y Doppler previo a la donación de sangre y posterior a la misma.

Asimismo, he sido informado de que se me realizarán estudios mediante ultrasonido, USCOM y ultrasonido Doppler cardiaco y vascular para la investigación en el conocimiento de cambios en el gasto cardiaco previo y posterior a la donación de sangre. Se me informó que el procedimiento a realizar no implica ningún riesgo para mí.

El estudio aportará información relacionada con el estado del corazón y del volumen sanguíneo y cómo se adapta el sistema circulatorio a los cambios de volumen inducidos por la extracción de sangre para la donación.

Estoy conforme con la intervención que se me ha propuesto. He leído y comprendido la información anterior. He podido preguntar y aclarar todas mis dudas. Por eso, he tomado consciente y libremente la decisión de autorizarla. También sé que puedo retirar mi consentimiento cuando lo estime oportuno.

Nombre y firma
de quien realiza el estudio

Testigo
Nombre y firma del donador 